\title{
A construction of left equalizer simple medial semigroups
}

\author{
Attila Nagy ${ }^{1}$ \\ Accepted: 25 August 2021 / Published online: 3 March 2022 \\ (c) The Author(s) 2022
}

\begin{abstract}
A semigroup $S$ is said to be left equalizer simple if, for all elements $a, b, x, y$ of $S, x a=x b$ implies $y a=y b$. It is known that left equalizer simplicity is a necessary condition for a semigroup to be embedded in a left simple semigroup. A semigroup satisfying the identity $a x y b=a y x b$ is called a medial semigroup. In this paper we show how to construct left equalizer simple medial semigroups, especially, medial semigroups which can be embedded in idempotent-free left simple semigroups.
\end{abstract}

Keywords Semigroup · Medial semigroup · Left simple semigroup · Left equalizer simple semigroup

Mathematics Subject Classification 20M10 · 20M30

\section{Introduction and motivation}

In [5, Theorem 1], P.M. Cohn gave necessary and sufficient conditions for a semigroup to be embedded in a left simple semigroup. Conditions differ essentially according to whether or not the semigroup contains an idempotent element (i.e. an element $e$ satisfying $e^{2}=e$ ). Cohn proved (see also the dual of [4, Theorem 8.19]) that a semigroup $S$ is embedded in an idempotent-free left simple semigroup if and only if $S$ is idempotent-free and satisfies the condition: for all $a, b, x, y \in S, x a=x b$ implies $y a=y b$. Using the terminology of [12], a semigroup $S$ satisfying this condition is called a left equalizer simple semigroup.

A semigroup is called a medial semigroup ( [2]) if it satisfies the identity $a x y b=a y x b$. Medial semigroups are well studied by many authors, see, for example, papers [1], [2], [6], [10], [17], and books [9], [14]. In the present paper, medial semigroups are also the subject of investigations. Using results of [12], we show how to construct left equalizer simple medial semigroups, especially medial semigroups which can be embedded in idempotent-free left simple semigroups.

Attila Nagy

nagyat@math.bme.hu

1 Department of Algebra, Institute of Mathematics, Budapest University of Technology and Economics, P.O. Box 91, Budapest 1521, Hungary 


\section{Preliminaries}

For the notions and notations not defined but used in this paper, we refer the reader to books [3], [4] and [9].

By [12, Definition 2.1], a semigroup $S$ is said to be left equalizer simple if, for every elements $a, b, x, y \in S, x a=x b$ implies $y a=y b$. By [5, Theorem 1], if a semigroup $S$ is embedded in a left simple semigroup, then $S$ is left equalizer simple.

In [12], we defined a construction, and showed how to get left equalizer simple semigroups by applying that construction. This construction plays an important role in our present investigation. Thus we cite it here.

Construction 2.1 [ [12, Construction 1]] Let $T$ be a left cancellative semigroup. For each $t \in T$, associate a nonempty set $S_{t}$ to $t$ such that $S_{t} \cap S_{r}=\emptyset$ for every $t, r \in T$ with $t \neq r$. For an arbitrary couple $(t, r) \in T \times T$ with $r \in t T$, let $\varphi_{t, r}$ be a mapping of $S_{t}$ into $S_{r}$ acting on the right. For all $t \in T, r \in t T, q \in r T \subseteq t T$, assume

$$
\varphi_{t, r} \varphi_{r, q}=\varphi_{t, q}
$$

On the set $S=\bigcup_{t \in T} S_{t}$ define an operation $*$ as follows: for arbitrary $a \in S_{t}$ and $b \in S_{x}$, let

$$
a * b=a \varphi_{t, t x} .
$$

If $a \in S_{t}, b \in S_{x}, c \in S_{y}$ are arbitrary elements then

$$
\begin{aligned}
a *(b * c) & =a * b \varphi_{x, x y}=a \varphi_{t, t(x y)} \\
& =a \varphi_{t, t x} \varphi_{t x, t(x y)}=a \varphi_{t, t x} * c=(a * b) * c .
\end{aligned}
$$

Thus $(S ; *)$ is a semigroup.

The semigroup $(S ; *)$ defined in Construction 2.1 is said to be a right regular extension of the left cancellative semigroup $T$. This extension is said to be injective if the mappings $\varphi_{t, r}$ are injective for every $t \in T$ and $r \in t T$. form.

Using the terminology of a right regular extension, [12, Theorem 2.2] has the following

Proposition 2.2 [ [12, Theorem 2.2]] A semigroup is left equalizer simple if and only if it is a right regular extension of a left cancellative semigroup. is

For a semigroup $S$, let $\theta_{S}^{\text {right }}$ denote the kernel of the right regular representation of $S$, that

$$
\theta_{S}^{\text {right }}=\{(a, b) \in S \times S:(\forall x \in S) x a=x b\} .
$$

By [11, Theorem 2], the $\theta_{S}^{\text {right }}$-classes of the semigroup $S=(S ; *)$ defined in Construction 2.1 are the sets $S_{t}(t \in T)$, and $S / \theta_{S}^{\text {right }} \cong T$. Thus an element $e \in T$ is an idempotent element if and only if $S_{e}$ is a subsemigroup of $(S ; *)$.

Proposition 2.3 Let $(S ; *)$ be a right regular extension of a left cancellative semigroup $T$, and let e be an idempotent element of $T$. Then the subsemigroup $S_{e}$ of $(S ; *)$ is a retract ideal extension of a left zero semigroup by a zero semigroup. 
Proof Since $S_{e}$ is a $\theta_{S}^{\text {right }}$-class of $S=(S ; *)$, the equality

$$
x * a=x * b
$$

holds for every $x, a, b \in S_{e}$. Let $a \in S_{e}$ be an arbitrary element. Then

$$
a^{2}=a * a=a * a^{2}=a^{3},
$$

and so

$$
\left(a^{2}\right)^{2}=a * a^{3}=a * a^{2}=a^{3}=a^{2},
$$

that is, $a^{2}$ is an idempotent element. Let $E\left(S_{e}\right)$ denote the set of all idempotent elements of $S_{e}$. For arbitrary $a, b \in S_{e}$, we have

$$
a * b=a^{2} \in E\left(S_{e}\right) .
$$

Thus $E\left(S_{e}\right)$ is an ideal of $S_{e}$, and the Rees factor semigroup $S_{e} / E\left(S_{e}\right)$ is a zero semigroup. For arbitrary $f_{1}, f_{2} \in E\left(S_{e}\right)$,

$$
f_{1} * f_{2}=f_{1} * f_{1}=f_{1} .
$$

Hence $E\left(S_{e}\right)$ is a left zero semigroup. For arbitrary $a, x \in S_{e}$, we have

$$
a \varphi_{e, e}=a * x \in E\left(S_{e}\right) .
$$

Thus $\varphi_{e, e}$ is a mapping of $S_{e}$ into $E\left(S_{e}\right)$. Let $a, b, x \in S_{e}$ be arbitrary elements. Then

$(a * b) \varphi_{e, e}=(a * b) * x=a *(b * x)=a *(x * b * x)=(a * x) *(b * x)=\left(a \varphi_{e, e}\right) *\left(b \varphi_{e, e}\right)$.

Hence $\varphi_{e, e}$ is a homomorphism of $S_{e}$ into $E\left(S_{e}\right)$. For every $f \in E\left(S_{e}\right)$, we have

$$
f=f * f=f \varphi_{e, e},
$$

that is, $\varphi_{e, e}$ leaves the elements of $E\left(S_{e}\right)$ fixed. Thus $\varphi_{e, e}$ is a retract homomorphism of $S_{e}$ onto the ideal $E\left(S_{e}\right)$. Hence $S_{e}$ is a retract ideal extension of the left zero semigroup $E\left(S_{e}\right)$ by the zero semigroup $S_{e} / E\left(S_{e}\right)$.

Applying also Proposition 2.3, we get the following corollary which will be used in the proof of Corollary 3.6.

Corollary 2.4 A right regular extension $(S ; *)$ of a left cancellative semigroup $T$ is idempotent-free if and only if $T$ is idempotent-free.

\section{Left equalizer simple medial semigroups}

In our investigation, two subclasses of semigroups play an important role. These are the class of left commutative semigroups and the class of right commutative semigroups. Using the terminology of [14], a semigroup is said to be left commutative if it satisfies the identity $x y b=$ $y x b$. The notion of a right commutative semigroup is defined analogously. We note that left (resp., right) commutative semigroups are also called left (resp., right) pseudo commutative semigroups (see, for example, [15], [18]). It is clear that every left commutative semigroup and every right commutative semigroup is medial.

Left commutative and right commutative semigroups appear in several papers, see, for example, [7], [8], [13], [15], [16], [18] and books [9], [14].

The next two lemmas are obvious consequences of the definitions. 
Lemma 3.1 On an arbitrary semigroup $S$, the following conditions are equivalent.

(1) $S$ is medial.

(2) The factor semigroup $S / \theta_{S}^{\text {right }}$ is left commutative.

Lemma 3.2 On an arbitrary semigroup $S$, the following conditions are equivalent.

(1) $S$ is left commutative.

(2) The factor semigroup $S / \theta_{S}^{\text {left }}$ is commutative.

In the next proposition, we show that the right cancellativity is a sufficient condition for a right commutative semigroup to be left equalizer simple. This fact and its dual will be used in the proof of Theorem 3.5.

Proposition 3.3 Every right cancellative right commutative semigroup is left equalizer simple.

Proof Let $a$ and $b$ be arbitrary elements of a right cancellative right commutative semigroup $S$. Assume $x a=x b$ for some $x \in S$. Then, for an arbitrary $y \in S$,

$$
y a x=y x a=y x b=y b x .
$$

Since $S$ is right cancellative, we obtain that

$$
y a=y b .
$$

Thus $S$ is left equalizer simple.

In the next proposition, we give a necessary and sufficient condition for a left equalizer simple semigroup to be right cancellative. The dual of this proposition will be used in the proof of Theorem 3.5 .

Proposition 3.4 Let $(S ; *)$ be a right regular extension of a left cancellative semigroup $T$. Then $(S ; *)$ is right cancellative if and only if $T$ is also right cancellative and the extension is injective.

Proof Assume that the left cancellative semigroup $T$ is also right cancellative and the mappings $\varphi_{t, r}(t \in T, r \in t T)$ are injective. To show that $(S ; *)$ is right cancellative, assume

$$
a * s=b * s
$$

for elements $a \in S_{x}, b \in S_{y}$ and $s \in S_{t}$. Then

$$
a \varphi_{x, x t}=b \varphi_{y, y t}
$$

from which it follows that

$$
x t=y t .
$$

As $T$ is right cancellative, we get

$$
x=y \text {. }
$$

Thus

$$
a \varphi_{x, x t}=b \varphi_{x, x t},
$$

from which we get $a=b$ by the injectivity of $\varphi_{x, x t}$. Thus $(S ; *)$ is right cancellative. 
Conversely, assume that $(S ; *)$ is right cancellative. First we show that the mappings $\varphi_{t, r}$ ( $t \in T, r \in t T$ ) are injective. Let $t \in T, r \in t T$ be arbitrary elements. Assume

$$
a \varphi_{t, r}=b \varphi_{t, r}
$$

for $a, b \in S_{t}$. Since $T$ is left cancellative, then there is exactly one $y \in T$ such that $r=t y$. Then, for an arbitrary $s \in S_{y}$, we have

$$
a * s=a \varphi_{t, r}=b \varphi_{t, r}=b * s .
$$

From the right cancellativity of $S$ it follows that $a=b$. Therefore, $\varphi_{t, r}$ is injective.

To show that $T$ is right cancellative, assume

$$
x t=y t
$$

for $x, y, t \in T$. Then, for arbitrary $a \in S_{x}, b \in S_{y}, s \in S_{t}$, we have

$$
a * s=a \varphi_{x, x t} \text { and } b * s=b \varphi_{y, y t} .
$$

As $x t=y t$, we get

$$
a * s, b * s \in S_{x t}
$$

As $S_{x t}$ is a $\theta_{S}^{\text {right }}$-class, we have

$$
s * a * s=s * b * s .
$$

By the assumption that $S$ is right cancellative, we get

$$
s * a=s * b,
$$

and so

$$
s \varphi_{t, t x}=s \varphi_{t, t y} .
$$

From this it follows that $t x=t y$. As $T$ is left cancellative, we get $x=y$. Hence $T$ is right cancellative.

The next theorem is the main result of the paper.

Theorem 3.5 A semigroup is left equalizer simple and medial if and only if it is a right regular extension of a semigroup which is an injective left regular extension of a commutative cancellative semigroup.

Proof Let $S$ be a left equalizer simple medial semigroup. By [12, Theorem 2.2] and Lemma 3.1, $S$ is a right regular extension of the left cancellative, left commutative semigroup $T=S / \theta_{S}^{\text {right }}$. By the dual of Proposition 3.3,T is right equalizer simple. By Lemma 3.2 and the dual of Proposition 2.2, $T$ is a left regular extension of the commutative, cancellative semigroup $U \cong T / \theta_{T}^{\text {left }}$. Since $T$ is left cancellative, then the dual of Proposition 3.4 implies that this left regular extension is injective. Hence $S$ is a right regular extension of the semigroup $T$ which is an injective left regular extension of the commutative cancellative semigroup $U$.

Assume that a semigroup $S$ is a right regular extension of a semigroup $T$ which is an injective left regular extension of a commutative cancellative semigroup $U$. First of all, we note that this assumption is correct, because the semigroup $T$ is left cancellative by the dual of Proposition 3.4. Thus the semigroup $S$ is left equalizer simple by Proposition 2.2. Since $U$ is a commutative semigroup and $U \cong T / \theta_{T}^{\text {left }}$, then the semigroup $T$ is left commutative by Lemma 3.2. As $T \cong S / \theta_{S}^{\text {right }}$, we get that the semigroup $S$ is medial by Lemma 3.1. 
Corollary 3.6 A semigroup is a medial semigroup which is embedded in an idempotent-free left simple semigroup if and only if it is a right regular extension of a semigroup which is an injective left regular extension of an idempotent-free commutative cancellative semigroup.

Proof It is obvious by [5, Theorem 1], Theorem 3.5, Corollary 2.4 and its dual.

Acknowledgements The author is thankful to the referee for the valuable suggestions which have improved the presentation of this article.

Funding Open access funding provided by Budapest University of Technology and Economics.

Open Access This article is licensed under a Creative Commons Attribution 4.0 International License, which permits use, sharing, adaptation, distribution and reproduction in any medium or format, as long as you give appropriate credit to the original author(s) and the source, provide a link to the Creative Commons licence, and indicate if changes were made. The images or other third party material in this article are included in the article's Creative Commons licence, unless indicated otherwise in a credit line to the material. If material is not included in the article's Creative Commons licence and your intended use is not permitted by statutory regulation or exceeds the permitted use, you will need to obtain permission directly from the copyright holder. To view a copy of this licence, visit http://creativecommons.org/licenses/by/4.0/.

\section{References}

1. C. Bonzini, A. Cherubini, Medial permutable semigroups. Semigroups, Szeged, Hungary. Proc. Coll. Math. Soc. János Bolyai 39, 21-39 (1981)

2. J.L. Chrislock, On medial semigroups. J. Algebra 12, 1-9 (1969)

3. A.H. Clifford, G.B. Preston, The Algebraic Theory of Semigroups I. (American Mathematical Society, Providence, Rhode Island, 1961)

4. A.H. Clifford, G.B. Preston, The Algebraic Theory of Semigroups II. (American Mathematical Society, Providence, Rhode Island, 1967)

5. P.M. Cohn, Embeddings in semigroups with one-sided division. J. London Math. Soc. 31(2), 169-181 (1956)

6. Z. Jiang, On medial local semigroups. Semigroup Forum 46, 120-122 (1993)

7. A. Nagy, Subdirectly irreducible right commutative semigroups. Semigroup Forum 46, 187-198 (1993)

8. A. Nagy, Right commutative $\Delta$-semigroups. Acta Sci. Math. (Szeged) 66, 33-46 (2000)

9. A. Nagy, Special Classes of Semigroups (Kluwer Academic Publishers, Dordrecht, 2001)

10. A. Nagy, Medial permutable semigroups of the first kind. Semigroup Forum 76-2, 297-308 (2008)

11. A. Nagy, Remarks on the paper "M. Kolibiar, On a construction of semigroups". Period. Math. Hung. 71(2), 261-264 (2015)

12. A. Nagy, Left equalizer simple semigroups. Acta Math. Hungar. 148(2), 300-311 (2016)

13. T.E. Nordahl, Finitely generated left commutative semigroups are residue finite. Semigroup Forum 28 , 347 (1984)

14. M. Petrich, Lectures in Semigroups (Akademie-Verlag, Berlin, 1977)

15. B.M. Schein, On the theory of restrictive semigroups. Izv. Vyssh. Uchebn. Zaved. Mat. No. 2, 152-154 (1963) (Russian)

16. B.M. Schein, Restrictive semigroups and bisemigroups (Technical Report. University of Arkansas, Fayetteville, Arkansas, USA, 1989), pp. 1-23

17. R. Strecker, Konstruktionen freier medialer Halbgruppen aus kommutativen Halbgruppen. Wiss. Zeitschr. PH Güstrow, math.-mat. Pak. 20, 265-271 (1982)

18. V.V. Vagner, Restrictive semigroups. Izv. Vyssh. Uchebn. Zaved. Mat. No 6, 19-27 (1962) (Russian)

Publisher's Note Springer Nature remains neutral with regard to jurisdictional claims in published maps and institutional affiliations. 